\title{
Electrical Excitation of the Acoustically Sensitive Auditory Nerve: Single-Fiber Responses to Electric Pulse Trains
}

\author{
Charles A. Miller, ${ }^{1,2}$ Paul J. Abbas, ${ }^{1,2}$ Barbara K. Robinson, ${ }^{1}$ Kirill V. Nourski, ${ }^{2}$ \\ FAWEN Zhang, ${ }^{1}$ AND FuH-ChERng Jeng ${ }^{2}$ \\ ${ }^{1}$ Department of Otolaryngology, University of Iowa Hospitals $\mathcal{E}$ Clinics, 21201 PFP, 200 Hawkins Drive, Iowa City, \\ IA 52242, USA \\ ${ }^{2}$ Department of Speech Pathology and Audiology, The University of Iowa, Wendell Johnson Speech and Hearing Center Iowa \\ City, IA 52242, USA
}

Received: 4 January 2006; Accepted: 17 March 2006; Online publication: 16 May 2006

\begin{abstract}
Nearly all studies on auditory-nerve responses to electric stimuli have been conducted using chemically deafened animals so as to more realistically model the implanted human ear that has typically been profoundly deaf. However, clinical criteria for implantation have recently been relaxed. Ears with "residual" acoustic sensitivity are now being implanted, calling for the systematic evaluation of auditory-nerve responses to electric stimuli as well as combined electric and acoustic stimuli in acoustically sensitive ears. This article presents a systematic investigation of single-fiber responses to electric stimuli in acoustically sensitive ears. Responses to 250 pulse/s electric pulse trains were collected from 18 cats. Properties such as threshold, dynamic range, and jitter were found to differ from those of deaf ears. Other types of fiber activity observed in acoustically sensitive ears (i.e., spontaneous activity and electrophonic responses) were found to alter the temporal coding of electric stimuli. The electrophonic response, which was shown to greatly change the information encoded by spike intervals, also exhibited fast adaptation relative to that observed in the "direct" response to electric stimuli. More complex responses, such as "buildup" (increased responsiveness to successive pulses) and "bursting" (alternating periods of responsiveness
\end{abstract}

Correspondence to: Charles A. Miller $\bullet$ Department of OtolaryngologyHead \& Neck Surgery, 21201 PFP - - UIHC • 200 Hawkins Drive, Iowa City, IA 52242, USA. Telephone: +1-319-3846758; fax: +1-3193846761; email: charles-miller@uiowa.edu and unresponsiveness) were observed. Our findings suggest that bursting is a response unique to sustained electric stimulation in ears with functional hair cells.

Keywords: auditory nerve, cat, cochlear implant, residual hearing, single fiber

\section{INTRODUCTION}

The criteria for cochlear-implant candidacy have recently been expanded to include individuals with significant "residual" low-frequency hearing, with promising results (von Ilberg et al. 1999; Gantz and Turner 2003). Animal models show that hair cells apical to implanted electrode arrays can survive over chronic periods ( $\mathrm{Ni}$ et al. 1992; Xu et al. 1997) and human studies indicate that acoustic sensitivity can be preserved after implantation (Kiefer et al. 1998). The standard animal model for cochlear implant research has long used a deaf cochlea to isolate the direct, electrical, depolarization of auditory nerve fibers (i.e., the " $\alpha$ " response of Moxon 1971). However, a new animal model is needed to study how electric stimulus coding is modified by viable hair cells, which may occur through several possible mechanisms.

\section{Electrophonic activation}

Moxon (1971) not only described the $\alpha$ response, but a $\beta$ response, in response to electric stimulation of hearing ears. It is attributed to electrically induced contraction of outer hair cells (OHCs), production of 
a traveling wave, and normal excitation of nerve fibers by inner hair cell (IHC) activity (Brownell et al. 1985; Nuttall and Ren 1995). As $\beta$ responses can occur coincidently with $\alpha$ responses (van den Honert and Stypulkowski 1984), they could result in relatively complex temporal responses.

\section{Spontaneous activity}

Modification of electric responses may not require activation of hair cells, but simply the existence of spontaneous activity. Such activity could modify $\alpha$ responses by placing fibers in states of partial refractoriness, thereby altering their responses (cf. Miller et al. 2001a).

\section{Efferent activity}

Efferent effects could involve the medial olivocochlear (MOC) or the lateral olivocochlear (LOC) systems, which project primarily to OHCs and peripheral axons of afferents, respectively (Warr and Guinan 1979). Although deafness would wipe out MOC functionality, LOC effects may persist if peripheral terminals remain. Thus, the normal cochlea may be influenced by both systems, whereas LOC effects may occur in deafened ears with peripheral processes.

\section{IHC depolarization}

IHCs may be excited through direct depolarization of their membranes, which presumably leads to the $\delta$ response, an infrequent response described by van den Honert and Stypulkowski (1984). IHC depolarization may also modulate spontaneous spike activity.

These mechanisms could conceivably improve electric stimulus coding or interfere with it. We recognize that electric hearing in acoustically sensitive ears may involve peripheral and central mechanisms; however, a clear understanding of effects at the level of the auditory nerve will not only elucidate peripheral mechanisms, but also improve the ability to interpret central measures.

In addition to the single-fiber studies mentioned above, von Ilberg et al. (1999) examined auditory nerve fiber responses to acoustic and electric stimuli, focusing primarily on the nerve's capacity to respond independently to both. In contrast, our study addresses how acoustic sensitivity alters fiber responses to electric stimuli, paralleling, to some degree, our earlier work that examined the electrically evoked compound action potential (ECAP) (Hu et al. 2003; Nourski et al. 2005). This study developed an acute animal model using a minimally invasive intracochlear electrode to preserve as much hearing as possible. We therefore sought not to model a human implant candidate with residual hearing, but to maximize the contrasts between deaf and hearing ears. Prior to addressing these goals, we characterized our animal preparations in terms of their ability to model acoustically sensitive ears, so that limitations could be identified. A moderate-rate ( 250 pulse/s) electric pulse train was used to examine a stimulus relevant to auditory prostheses while providing for the ability to examine both the $\alpha$ and $\beta$ responses. Another effort, to be reported in a subsequent article, will address how combined acoustic and electric stimuli modify single-fiber responses evoked by electric stimuli.

\section{METHODS}

Animal preparation

Adult cats free of external or middle ear infection were used in acute experiments. The surgical techniques have been previously described (Miller et al. 2001b); information on animal maintenance and deviations from previously published procedures are described here. All animal-use protocols were approved by the University of Iowa Institutional Animal Care and Use Committee and adhered to the regulations of the United States National Institutes of Health.

Cats were first sedated by an intramuscular injection of ketamine $(22 \mathrm{mg} / \mathrm{kg})$ and xylazine $(1.1 \mathrm{mg}$ / $\mathrm{kg}$ ), after which intravenous Nembutal was administered $(8-13 \mathrm{mg} / \mathrm{kg})$ at intervals as dictated by reflex and vital signs. Atropine sulfate $(0.04 \mathrm{mg} / \mathrm{kg} / 8 \mathrm{~h}$, s.c. $)$ was given to reduce mucosal secretions and dexamethasone $(1.0 \mathrm{mg} / \mathrm{kg} / 12 \mathrm{~h}$, i.v.) was used to control edema. Animal respiration was maintained by tracheal intubation and delivery of oxygen through a Harvard Apparatus ventilator (12-15 ml tidal volume, 45-60 cycle $/ \mathrm{min})$. Body temperature was stabilized via a circulating warm-water pad and insulating drapes. Core temperature, blood oxygen saturation, and heart rate were continuously monitored with a Pace Tech 4000B vital signs monitor, and expired $\mathrm{CO}_{2}$ was monitored using a BCI Industries capnometer. Animals were maintained at surgical levels of anesthesia for the duration of the experimental session. The state of anesthesia was assessed by observing body temperature, heart rate, and respiration patterns, as well as pinch reflexes. At the conclusion of data collection, the animal was euthanized by an overdose of anesthesia.

After surgical exposure of the auditory nerve, the bulla was opened to provide cochlear access. A 0.4 - to 0.5-mm diameter $\mathrm{Pt} / \mathrm{Ir}$ ball electrode was positioned near the round window margin against the bone of the cochlea to provide a means of recording the com- 
pound action potential (CAP) to assess acoustic sensitivity. A small cochleostomy was made at a point approximately 1-2 $\mathrm{mm}$ medial to the round window by using a $0.5-\mathrm{mm}$ diamond burr. A Teflon-coated $\mathrm{Pt} /$ Ir wire (3 mil wire diameter; A-M Systems, Inc.), with a 0.3-mm-diameter flame-balled end, was inserted into the defect to a depth of approximately $1.5 \mathrm{~mm}$, providing the current path for monopolar intracochlear stimulation of the basal aspect of the scala tympani.

\section{Stimuli}

All stimuli were generated using custom software (based on the Labview platform) that controlled an Instrutech Corporation ITC-18 data acquisition board. Waveforms were represented by 16-bit integers, and samples were output at a rate of 100,000 sample/s. Acoustic clicks, used for assessing hearing sensitivity, were generated using $100 \mu \mathrm{s} /$ phase biphasic pulses delivered to a Beyerdynamics DT48 earphone coupled to the external canal by a speculum. The pulse delivery rate was $30 \mathrm{pulse} / \mathrm{s}$. The acoustically evoked CAP was recorded before and after the cochleostomy and insertion of the stimulating electrode to assess surgical and insertion trauma. All electric stimuli were in the form of $40 \mu \mathrm{s} /$ phase biphasic pulses fed to a custommade current source with optical isolation and a capacitively coupled output. The preliminary ECAP measures were evoked using single pulses presented at a relatively low (30 pps) rate. Level was varied up to that which produced a maximum (saturated) ECAP amplitude or $2.0 \mathrm{~mA}$, whichever was lower. The same low-rate stimulus was used to present a continuous train of pulses as the single-unit search stimulus, with its initial level determined by that which evoked an ECAP amplitude $80-90 \%$ of the maximum amplitude recorded.

For single-fiber data collection, the electric stimuli consisted of trains of $40 \mu \mathrm{s} /$ phase biphasic pulses presented at $250 \mathrm{pulse} / \mathrm{s}$ for a train duration of 300 ms. That rate was chosen to be comparable to those used in clinical devices, yet limited so that both $\alpha$ and $\beta$ responses could be recorded in the postpulse intervals. Once a fiber was encountered, responses were recorded by using a current level chosen to evoke a first-pulse response probability of about $75 \%$ or greater. This was done to rapidly record responses to the train of pulses in the presence of expected adaptation. Fiber holding-time permitting, stimulus level was then varied in approximately linear current steps to explore the fiber's dynamic range. We typically presented $30-40$ pulse trains to obtain histograms and compute response statistics such as firing efficiency (FE) and jitter.

As noted above, this study was part of a larger effort that examined electric responses as well as responses to combined electric and acoustic stimuli. To that end, the aforementioned electric train stimuli were interleaved with two other stimuli: (1) the same 300-ms electric train presented along with a 100-ms acoustic noise burst (turned on $50 \mathrm{~ms}$ after train onset) and (2) the 100-ms acoustic noise burst presented by itself. The noise burst was created by a wide-band (20-100 kHz) Grason-Stadler model 455C white noise generator and was presented at levels typically between 90 and $100 \mathrm{~dB}$ SPL (rms equivalent). Silent intervals of $700 \mathrm{~ms}$ were used between each of these three stimuli to reduce the influence of long-term adaptation.

Collection of data for one histogram in response to an electric pulse train required about $2.2 \mathrm{~min}$. Time permitting, acoustic, frequency-modulated sinusoidal sweeps were used to obtain estimates of characteristic frequency. This involved a two-step process in which a wide sweep $(1 \mathrm{~Hz}-30 \mathrm{kHz})$ was used, followed by a narrower sweep encompassing $2-5 \mathrm{kHz}$, while tracking level downward in 10-dB steps to find threshold responses.

\section{Response recording}

Acoustic sensitivity of the preparation was assessed by visually determining the threshold response of the clickevoked CAP. These recordings, made using the roundwindow electrode, were amplified $(100 \times)$ and low-pass filtered $(30 \mathrm{kHz}, 120 \mathrm{~dB} /$ decade) prior to digital acquisition at 100,000 sample/s and 16-bit resolution.

ECAP growth functions produced by single $40 \mu \mathrm{s} /$ phase biphasic current pulses were obtained to assess the sensitivity of each subject to electric stimulation. Stimulus levels corresponding to $85-90 \%$ of the ECAP maximum (saturation) amplitude were typically used for search levels for single-fiber recordings. ECAPs were recorded using two $\mathrm{Pt} / \mathrm{Ir}$ ball electrodes; one ball positioned on the nerve trunk and the other $2 \mathrm{~mm}$ above the nerve, with both bathed with $0.9 \%$ saline solution. ECAP potentials were amplified and filtered in the same process used to collect CAPs.

Single-fiber action potentials were measured using standard micropipette techniques (cf. Miller et al. 1999). Potentials were amplified $(10 \times)$ and low-pass filtered (10 kHz cutoff; $40 \mathrm{~dB} /$ decade) by an Axon Instruments Axoprobe headstage and amplifier, followed by additional filtering $(100 \mathrm{~Hz}$ high pass, $40 \mathrm{~dB}$ decade and $20 \mathrm{kHz}$ low pass, $120 \mathrm{~dB} /$ decade). Response waveforms were acquired by the ITC-18 system using 16-bit representation and a sampling rate of $50,000 \mathrm{sample} / \mathrm{s}$.

\section{Data analysis and reduction}

All single-fiber traces were stored for off-line analysis using routines written with Matlab (Natick, MA, USA; 
version 6.1). These routines performed waveform "template" subtractions (cf. Miller et al. 1999) used to reduce stimulus and ECAP artifacts. As responses to pulse trains were recorded, the routines for artifact reduction were somewhat more complex, requiring the generation of separate templates for each pulse of the train. Spike detection was based upon achieving a criterion amplitude and spike latency was defined as the time between the pulse onset and peak of the spike. Although this procedure was largely automated, we routinely examined the computed spike "picks" visually as a check of the software routines. Although labor-intensive, this added step provided a higher degree of confidence in our spike picks.

We computed $\mathrm{FE}$ as the ratio of the number of spikes elicited and the number of times a pulse was presented. That is, FE was computed for each pulse in the electric train. Jitter, the temporal uncertainty of spike timing, was computed as the standard deviation of the spike times, again on a per-pulse basis. Characterization of the $\beta$ response required two steps. First, its presence in the response of a fiber was assessed by inspection of poststimulus time (PST) histograms; two modes corresponding to the $\alpha$ and $\beta$ responses (Moxon 1971) had to be identified. We then used separate temporal analysis windows $(0.2-1.0$ and $1.2-$ $3.5 \mathrm{~ms}$ ) to evaluate both the $\alpha$ and $\beta$ response. The use of these analysis windows precluded the separate analysis of the infrequent $\delta$ responses (van den Honert and Stypulkowski 1984). Spontaneous rates (SRs) were estimated over prestimulus intervals totaling 4-6 s of sampled time. Some of our analyses required the determination of the presence or absence of a particular response (such as a $\beta$ response). To that end, a binary $(0 / 1)$ variable was used to associate such phenomena with a fiber. Because the size of the data sets (i.e., number of files) for each fiber varied considerably, sampling bias could influence the statistics obtained from such binary "indicator" variables. To control for such bias, we required minimumsize data sets, typically by accepting data only from fibers that yielded data from a minimum of three electric stimulus levels. Such fiber selection rules are explicitly noted in the text.

\section{RESULTS}

The data presented in this report were obtained from a total 264 fibers from 17 cats. Not all fibers contributed to each data set, due primarily to variability in fiber contact time. Specific data set sizes are noted for individual analyses. In three of our first four preparations, acoustic threshold shifts (assessed either by the single-fiber threshold shift relative to the
Liberman and Kiang 1978 high-SR threshold curve or our repeated click-evoked CAP measures) were greater than $40 \mathrm{~dB}$. Threshold shifts in subsequent experiments were lower, as shown below. The acoustic threshold shift due to insertion of the intracochlear electrode was small (about 10-15 dB), although progressive shifts were often observed over the 12- to 18-h periods of data collection. As noted earlier, the collection of pulse-train responses, with all stimulus conditions fixed, required a relatively long time $(\sim 2 \mathrm{~min})$. Whenever we could, we pooled data across as many fibers as possible, in order to provide statistical descriptions of fiber response properties.

\section{The animal model}

Figures 1, 2, and 3 summarize basic acoustic properties of fibers from 14 cats that yielded the most acoustic threshold data. All data were obtained after

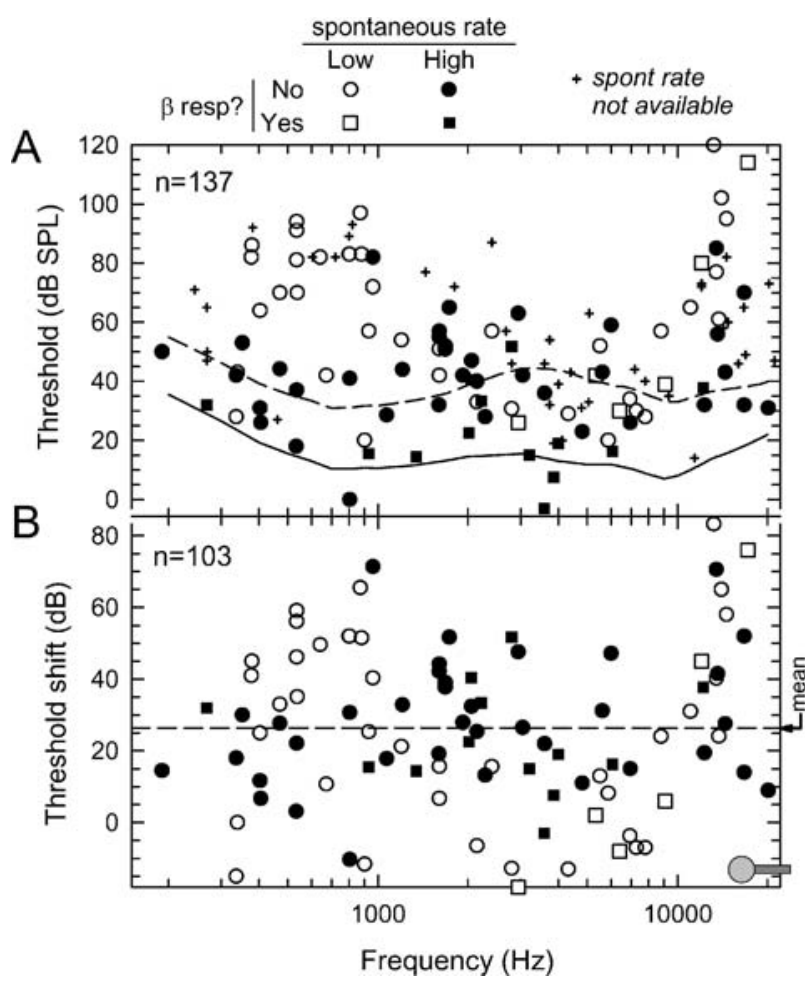

FIG. 1. Summary of basic acoustic properties of fibers of this study. (A) Acoustic thresholds and best frequencies for 137 fibers. Symbol types classify each fiber as low or high SR and whether or not electric stimulation evoked an identifiable $\beta$ response. Two contour lines are shown. The solid contour represents the mean thresholds for high-SR fibers of normal-hearing cats (Liberman and Kiang 1978), whereas the dashed line indicates mean thresholds for lowSR fibers of normal-hearing cats, computed using the low-SR correction data of Liberman (1978). (B) Acoustic threshold shifts computed using these two threshold contours. The dashed horizontal line indicates the mean threshold shift $(26.4 \mathrm{~dB})$. The approximate location of the stimulating electrode is indicated by the symbol in the bottom right of the graph. 

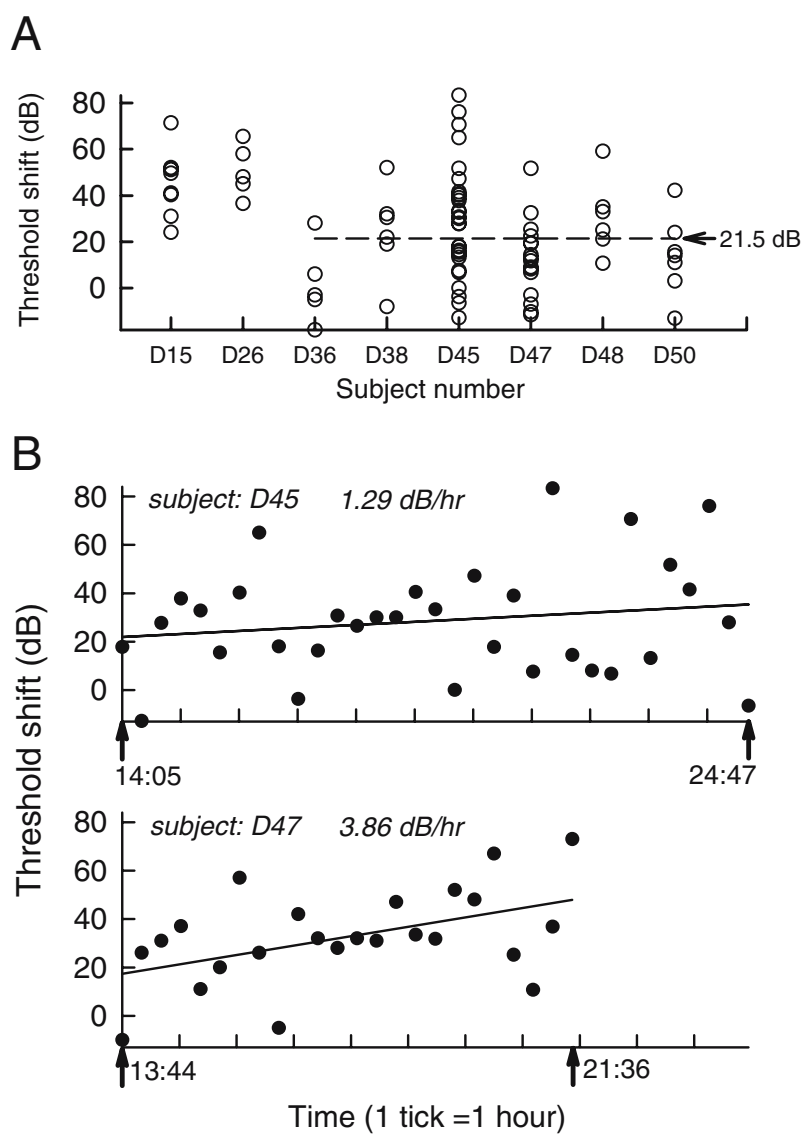

FIG. 2. Examination of within- and across-animal variation of fiber acoustic threshold shifts. Shifts were computed in the manner used for Fig. 1. (A) Threshold shifts for all cats that yielded acoustic data for at least five fibers. The mean shift for the last six cat experiments was $21.5 \mathrm{~dB}$. (B) Acoustic threshold shift versus time of data acquisition for fibers of the two cats (D45, D47) that yielded the most data. The arrows along the abscissa indicate the data collection starting time and completion time in hours and minutes. The data are fit to linear regression lines.

cochlear implantation. Acoustic thresholds vs. characteristic frequencies are shown in Figure 1A, along with indications of the presence or absence of electrophonic responses (indicated by symbol shape) and assignment of each fiber into low and high SR categories (indicated by filled or unfilled symbols). The SR groups were defined using a boundary rate of 25 spike/s, consistent with previous work (Liberman 1978; Liberman and Kiang 1978). Mean thresholds for high-SR fibers from the cats of Liberman and Kiang (1978), identified as "normal," but not reared under special, acoustically controlled, conditions, are indicated by the solid line in Figure 1A. Using threshold corrections for low-SR fibers (Liberman 1978), mean thresholds for low-SR fibers of normal cats are also shown (dashed line). The symbol at the bottom right of the figure shows the approximate location of the stimulating electrode, estimated from measurements of cochlear dissections and relating them to the place-frequency map of Liberman (1982).

Figure 1B replots the threshold data as threshold shifts. Shifts were computed relative to either the aforementioned high-SR threshold curve of Liberman and Kiang (1978) or the corrected curve for low-SR fibers (Liberman 1978). Across all fibers, the mean threshold shift was $26.4 \mathrm{~dB}$. Our limited data set makes it difficult to discern trends across frequency. To a first approximation, shifts appear to be uniformly distributed across frequency, with the possible exception of larger shifts at the most basal sites. Mean threshold shifts may be somewhat greater for fibers relatively close to the site of the intracochlear electrode.

Figure 1 also indicates that several fibers exhibited electrophonic $(\beta)$ responses, as determined by a second mode in the PST histogram (examples of $\beta$ responses can be seen in the histograms of Figure 6, which will be discussed later). To assess the incidence of this response, we surveyed all fibers from which electric responses were recorded from at least three levels. Of that pool of 94 fibers, $23(24.5 \%)$ had a mode that corresponded to a $\beta$ response, higher than the $13 \%$ incidence reported by van den Honert and Stypulkowski (1984). Figure 1 demonstrates that $\beta$ responses can occur in fibers over a wide range of characteristic frequencies, a finding similar to that reported by Javel and Shepherd (2000).

Figure 2 presents threshold shifts both across (Fig. 2A) and within (Fig. 2B) experiments. In Figure 2A, shifts are plotted for eight cats in the order in which the experiments were conducted, encompassing all subjects that yielded acoustic threshold for five or more fibers. Significant variability in threshold shifts is evident within and across subjects. Discounting the

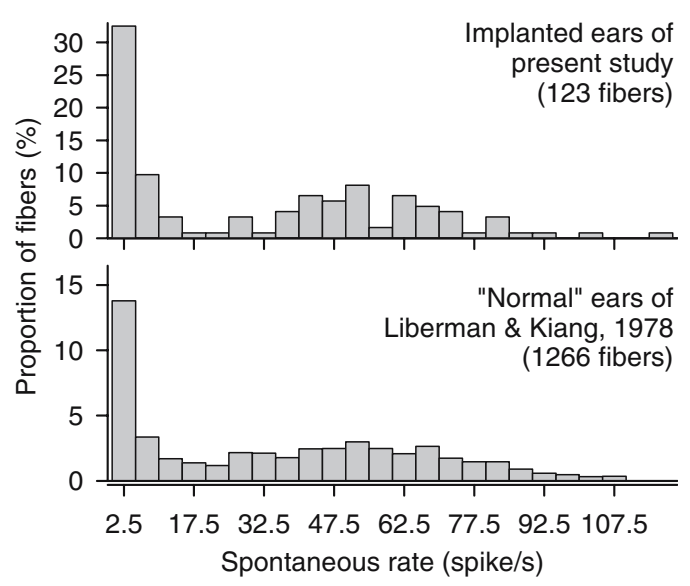

FIG. 3. Spontaneous rate histograms for the fibers of this study (top plot) and the fibers of the Liberman and Kiang (1978) study (bottom plot). 
data from the first two cats (subjects D15 and D26), the mean threshold shift was $21.5 \mathrm{~dB}$, suggesting possible improvement in experimental skill. Reduced sensitivity was observed over the course of most experiments, as seen in Figure 2B, where shifts are plotted vs. time for two cats that yielded the most threshold data. Linear regressions over these sets suggest increases over time: the rate of increase was $1.3 \mathrm{~dB} / \mathrm{h}$ for $\mathrm{D} 45$ and $3.9 \mathrm{~dB} / \mathrm{h}$ for $\mathrm{D} 47$. In the case of $\mathrm{D} 45$, the increase was not statistically significant $\left(r=0.18, n=33, p_{\text {error }}>0.1\right)$, whereas in the case of $\mathrm{D} 47$, it was $\left(r=0.48, n=24, p_{\text {error }}<0.05\right)$, using the test of the significance of the linear correlation (Bevington 1969).

Figure 3 compares an SR histogram from our study and that obtained by Liberman and Kiang (1978) from cats with normal acoustic sensitivity. Both distributions span comparable ranges of SRs and demonstrate bimodality. However, our distribution shows a bias toward lower rates. Using a 25 spike/s cutoff for low-SR fibers, we found that $47 \%$ of the fibers from implanted ears were low-SR units, whereas only $21 \%$ of the fibers from Liberman and Kiang's (1978) study fell in that category. This is consistent with observations of lower mean SRs from cats with trauma, as observed across cats raised in low-noise environments, "normal" cats, and cats with noiseinduced hearing losses (Liberman 1978; Liberman and Kiang 1978).

From these data, we conclude that our animal model preserves important properties of the normal, acoustically sensitive ear, but not without some changes associated with cochlear trauma. In general, these changes are moderate. Important properties such as acoustic sensitivity across the cochlea, a wide range of SRs, and demonstration of electrophonic responses are preserved. Although mean thresholds can increase over the course of the acute experiments, this increase can be relatively small.

\section{Fiber response properties}

Long-term stability and slow adaptation. An assumption made in computing PST histograms is that response characteristics are constant across the repeated measures, or sweeps. Although moderate-rate pulse trains were used in this study, it is possible that they could cause slow adaptation, or fatigue, across sweeps. We therefore examined spike probability (or FE) as a function of sweep number. Exemplar data from four fibers are shown in Figure $4 \mathrm{~A}$ for different stimulus levels. Each datum represents the mean FE across one pulse-train presentation, with $35-40$ sweeps in each plot. The functions in the first two columns are representative of typical patterns, whereas those of the last two columns show extreme
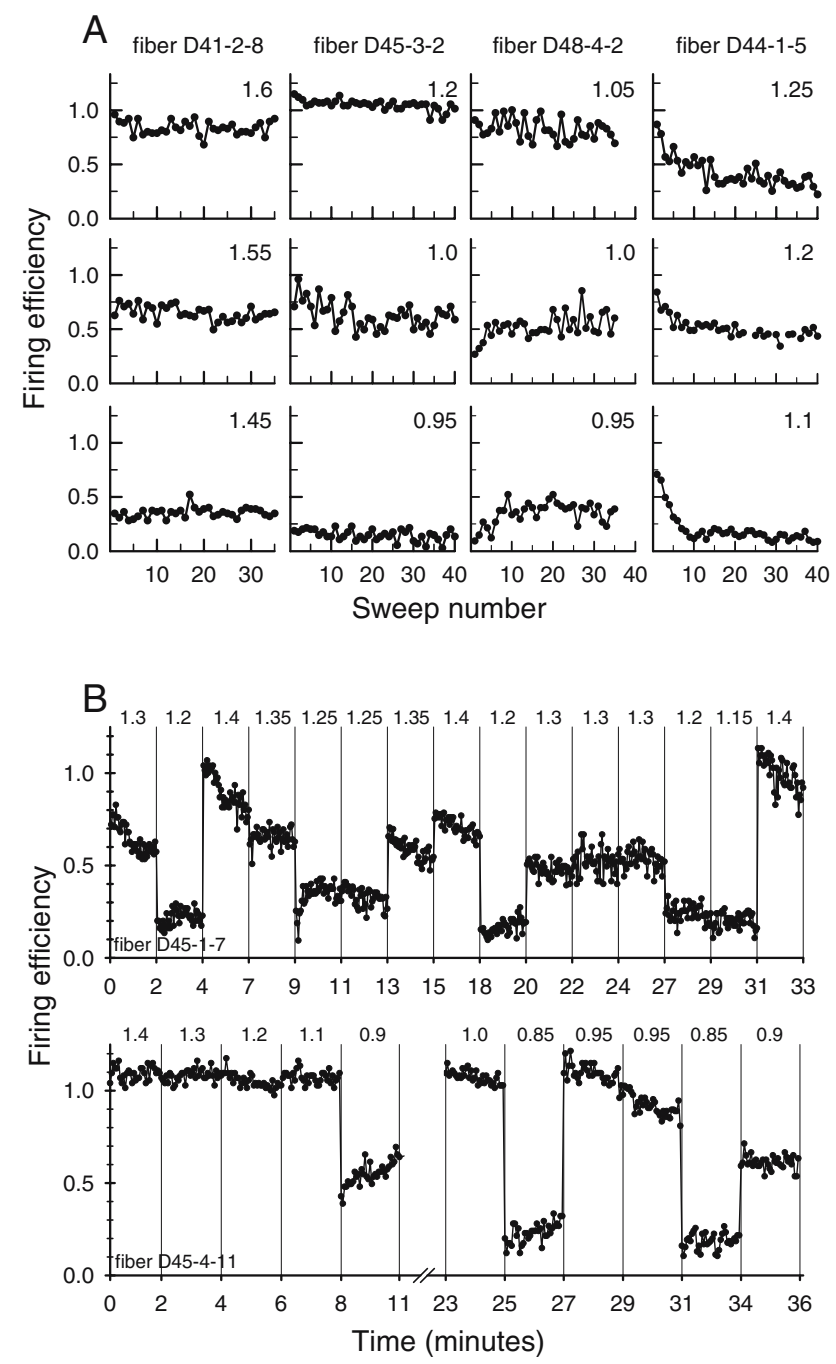

FIG. 4. (A) Firing efficiency (FE) versus sweep number for four fibers and three stimulus levels per fiber. For each sweep, FEs were computed by collapsing fiber activity across the entire epoch of each 300-ms pulse train. The number in the top right corner of each graph indicates stimulus level in mA. (B) Response probabilities for two fibers over the entire time of data collection. Vertical lines separate data for each file (i.e., set of repeated pulse trains) and the numbers at the top of each graph indicate stimulus level in $\mathrm{mA}$.

cases of either increases (third column) or decreases (fourth column) in responsiveness. Such unusual changes were observed in less than $15 \%$ of the fibers. To quantify the overall change in responsiveness, the plots such as those of Figure 4A were fit by linear regression for 21 fibers. The resultant slopes were first averaged within each fiber (across stimulus levels) before computing a grand (across-fiber) average. Across either 35 or 40 repeated sweeps, the mean $\mathrm{FE}$ change was $-8.4 \%$. We concluded that this was an acceptably low level of slow adaptation for deriving PST histograms.

Although this study was not designed to assess long-term or slow adaptation, we noted changes in fiber responsiveness over durations of data collection 
spanning many minutes. Figure 4B presents the mean (across train) FEs for two fibers that were held for more than $30 \mathrm{~min}$. Vertical lines separate the data from each file, each of which comprised 30-40 repeated train presentations. The stimulus level (in $\mathrm{mA}$ ) used in each case is shown along the top of each graph. The data from fiber D45-1-7 (upper graph) exhibited an unusually high degree of within-file adaptation, whereas that of D45-4-11 (lower graph) demonstrated greater (and more typical) within-fiber stability, as noted in the discussion of the plots in Figure $4 \mathrm{~A}$.

Although within-file adaptation is seen in the two plots of Figure 4B, there was generally a high degree of across-file stability in the fibers' responses. This is observed by comparing the mean FEs across time for the various stimulus levels across the two plots of Figure 4B. The data also suggest that adaptation depends on stimulus level. At relatively high stimulus levels, "saturated" response rates could be maintained across the repeated train presentations, as seen in the lower plot of Figure 4B for the 0- to 8-min epoch. However, at moderate stimulus levels, adaptation occurred in both fibers (at levels of 1.35-1.4 mA for fiber D45-1-7 and at levels of 0.95-1.0 mA for fiber D45-4-11). Adaptation is not evident for the lowestlevel responses, such as those in the $1.15-1.25 \mathrm{~mA}$ range for fiber D45-1-7 and those in the $0.85-0.9 \mathrm{~mA}$ range for fiber D45-4-11.

Threshold and dynamic range. Threshold and dynamic range of electrically stimulated fibers were estimated from a pool of fibers that provided histograms at four or more current levels while demonstrating no significant adaptation across data sets (which would confound the measures). A total of 27 fibers from seven cats fulfilled those criteria. Threshold, defined as the level eliciting a $50 \% \mathrm{FE}$, was typically estimated by linear interpolation of the FE vs. level function. Dynamic range, defined as the current range spanning FEs of 10-90\%, was also typically estimated by interpolation (or extrapolation) of the $\mathrm{FE}$ vs. level function. Measures were based upon the responses to the first pulse of each train to allow comparison with published data.

Over the 27 fibers, the mean threshold and dynamic range were $1.17 \mathrm{~mA}$ and $1.2 \mathrm{~dB}$, respectively. Figure 5 presents the two data sets and compares them against a previous study of feline fibers from chemically deafened cochleae (Miller et al. 2001b). Using the same $40 \mu \mathrm{s} /$ phase biphasic pulses and a similar monopolar electrode positioned in the basal turn of the scala tympani, that study reported a mean threshold and dynamic range of $0.85 \mathrm{~mA}$ and 1.06 $\mathrm{dB}$, respectively. A $t$-test conducted across the two sets of threshold data indicated that the acoustically sensitive fibers had significantly higher thresholds

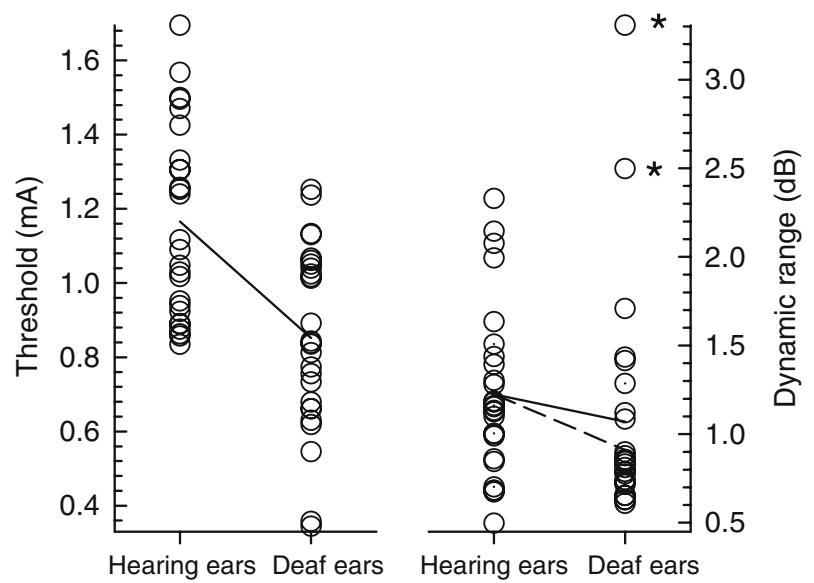

FIG. 5. Comparison of fiber thresholds (left panel) and fiber dynamic ranges (right panel) from the "hearing ears" of this study and the "deaf ears" from the study of Miller et al. (2001b). The solid line segments indicate linear regression results; the dashed line represents the linear regression obtained after removing the top two points indicated by asterisks.

$\left(t=4.63, p_{\text {error }}=0.000025 ; d f=52\right)$. A $t$-test across the sets of dynamic range data failed to reach significance $\left(t=0.83, p_{\text {error }}=0.41, d f=51\right)$. However, removal of the two highest values in the "deafened" set of data (see data labeled with asterisks in Fig. 5) resulted in a statistically significantly greater dynamic range for the acoustically sensitive fibers $(t=2.38$, $p_{\text {error }}=0.021, d f=49$ ).

Relationship between the $\alpha$ and $\beta$ responses. As previously reported, the direct $(\alpha)$ and electrophonic ( $\beta$ ) responses are readily identified in the PST histogram by separate, well-defined modes (van den Honert and Stypulkowski 1984; Javel and Shepherd 2000). Histograms from a fiber with both responses are shown in Figure 6; these are "period histograms" in that the spike times were computed from the onset of the previous pulse in the train and cover the 4-ms interval between successive pulses. Histograms are shown for three electric levels and three different 20-ms epochs of a 150-ms train.

From the histograms, it is clear that the direct and electrophonic responses demonstrate differing degrees and rates of adaptation to the 250 pulse/s train. Figure 7 shows typical trends of how $\alpha$ and $\beta$ response probabilities change across the duration of the pulse train for two fibers for which several current levels were evaluated. Over the 300-ms pulse train, the $\beta$ response exhibits relatively fast adaptation, whereas the $\alpha$ response demonstrates characteristically little adaptation. As has been reported elsewhere, the $\beta$ response dominates at lower electric levels, whereas the $\alpha$ response dominates at higher levels. The $\beta$ response decay rates seen here are typical of what was observed in eight fibers in which robust $\beta$ responses 
were collected across multiple levels. Across those fibers, the mean value of the time constant for $\beta$ response decay was $17.8 \mathrm{~ms}$, with a standard deviation of $7.0 \mathrm{~ms}$.

Our limited data suggest that the rate of $\beta$ adaptation depends more on stimulus level than on the $\alpha$ response rate. In the fibers shown in Figure 7, it appears that the addition of the $\alpha$ response at higher levels has little refractory effect on the firing probability of the $\beta$ response, an observation consistent with the results reported by van den Honert and Stypulkowski (1984). Finally, as shown in the lower left panels of Figure 7, it is possible to maintain a relatively constant, although partially adapted, $\beta$ response across the pulse train by using lower stimulus levels that evoke lower FEs to the first pulse.

The presence of $\alpha$ and $\beta$ responses would be expected to influence the spectral information transmitted by a fiber's firing pattern. To explore this, the fast Fourier transforms (FFTs) of the period histograms of fiber D41-2-6 (Fig. 6) were computed. To capture the effects of the fast-adapting $\beta$ response on the temporal code, the FFTs were based on period histograms derived by averaging together the histograms from the first four stimulus periods, i.e., the

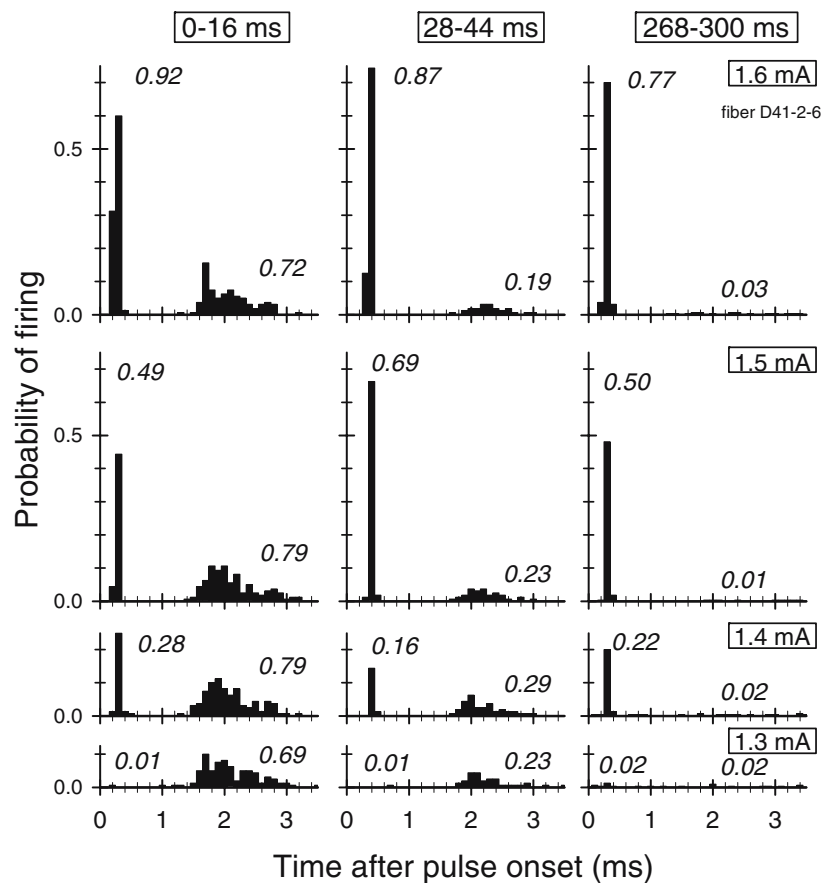

FIG. 6. Period histograms for a fiber exhibiting both "direct" $(\alpha)$ responses and electrophonic ( $\beta$ ) responses, as evidenced by two distinct modes. Each row corresponds to a different stimulus level (indicated along the right margin of the figure) and each column corresponds to a different analysis window (indicated at the top of each column). The histograms indicate that the $\beta$ response is dominant at the lowest levels and prone to relatively fast adaptation. The two numbers (in italics) shown in each graph indicate the firing efficiency of the $\alpha$ and $\beta$ responses.

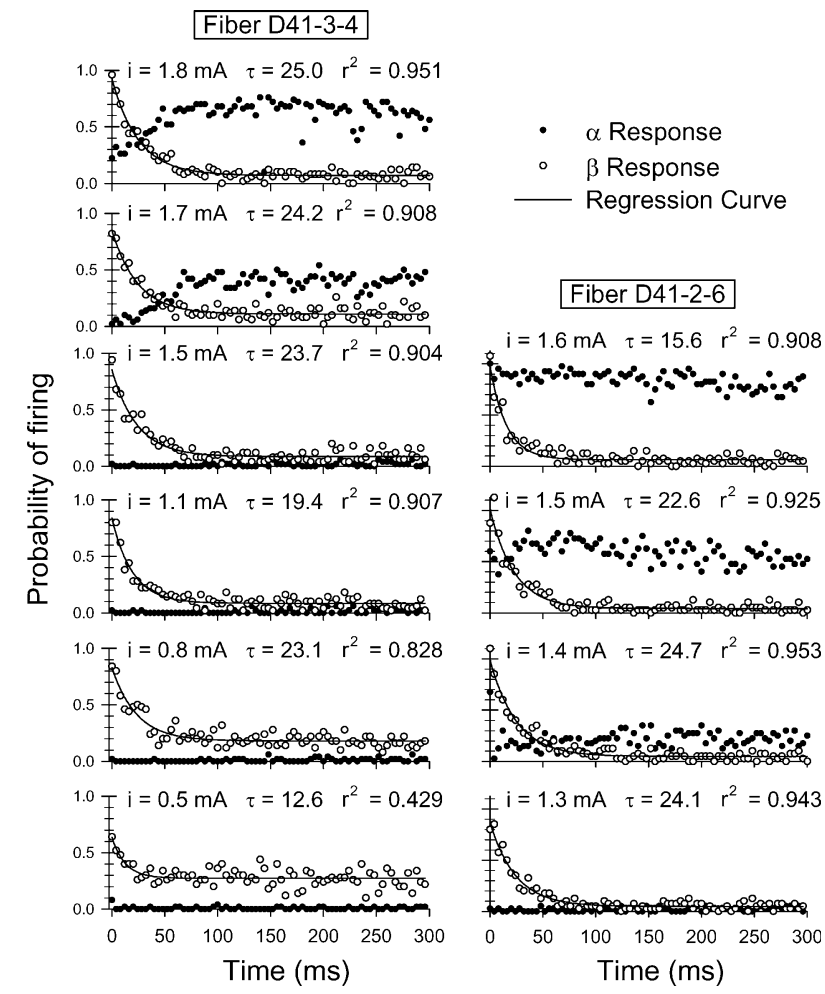

FIG. 7. Poststimulus time (PST) histograms for two fibers that exhibited both a direct response (filled symbols) and an electrophonic response (open symbols). Each electrophonic curve is fit to a decaying exponential curve. Along with the stimulus current level, the fitted time constant $(\tau)$ and coefficient of determination $\left(r^{2}\right)$ are shown in each panel.

first $16 \mathrm{~ms}$ of the response following stimulus onset. To reduce spectral splatter and improve resolution, 25 copies of each 80-point (i.e., $4 \mathrm{~ms}$ ) histogram were concatenated prior to performing the FFT. Resultant spectra are shown in Figure 8. At the lowest stimulus level $(i=1.3 \mathrm{~mA})$, the greater temporal dispersion of the dominant $\beta$ response results in a relatively strong representation of the fundamental $(250 \mathrm{~Hz})$ component of the stimulus, whereas at higher levels, the highly synchronized and dominant $\alpha$ response results in spectra dominated by higher harmonics. Given the relatively fast adaptation of the $\beta$ response, large spectral changes would also occur as a function of time across the pulse train.

Jitter. Jitter was computed using spike times that occured over a 3.5-ms window immediately after offset of the stimulus pulse. Figure 9A shows how jitter varies with FE for a total of 118 fibers, with fibers grouped into low and high SR categories. Jitter is correlated with FE (or stimulus level), but it is a weaker relationship than the one observed for SR. Electrophonic responses (filled symbols, Fig. 9A) are shown to elevate jitter. The relationship between jitter and SR is explicitly shown in Figure 9B, where 
jitter (as assessed for a firing efficiency of 50\%) is plotted vs. SR. Also shown in this panel are the mean jitter values for deafened fibers (Miller et al. 2001b). This comparison shows that jitter is approximately 1 order of magnitude greater in ears with acoustic sensitivity than in deaf ears.

Changes across the pulse train. Figure 10 presents PST histograms for four fibers chosen to represent the available data. In each case, data are shown for four stimulus levels. Histograms of the top half were derived using $50-\mu$ s bin widths, whereas those of the lower half were made using wide $(4 \mathrm{~ms})$ bins. The lower plots also indicate jitter estimates for bins containing at least five spikes. The plotted fibers were chosen for a range of acoustic sensitivities, SRs, and the presence or absence of an electrophonic response. The fibers shown in Figure 10 demonstrate moderate levels of rate adaptation, a typical finding. Also typical is the trend for this adaptation to be abolished at higher stimulus levels, as is seen in the histograms of columns 1, 2, and 4. We observed similar level-dependent adaptation in 18 of 28 fibers from which histograms were obtained at low, middle,

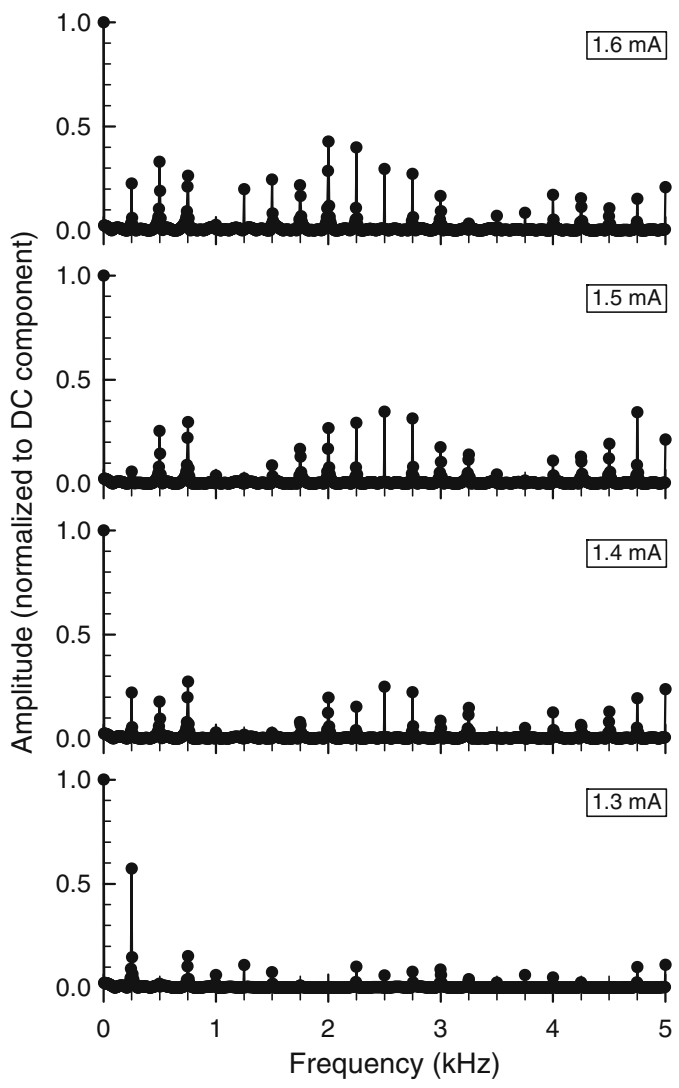

FIG. 8. Frequency spectra of the interval histograms of a fiber (D41-2-6) exhibiting both a direct $(\alpha)$ and an electrophonic $(\beta)$ response. Fast Fourier transform spectra were computed as described in the text.
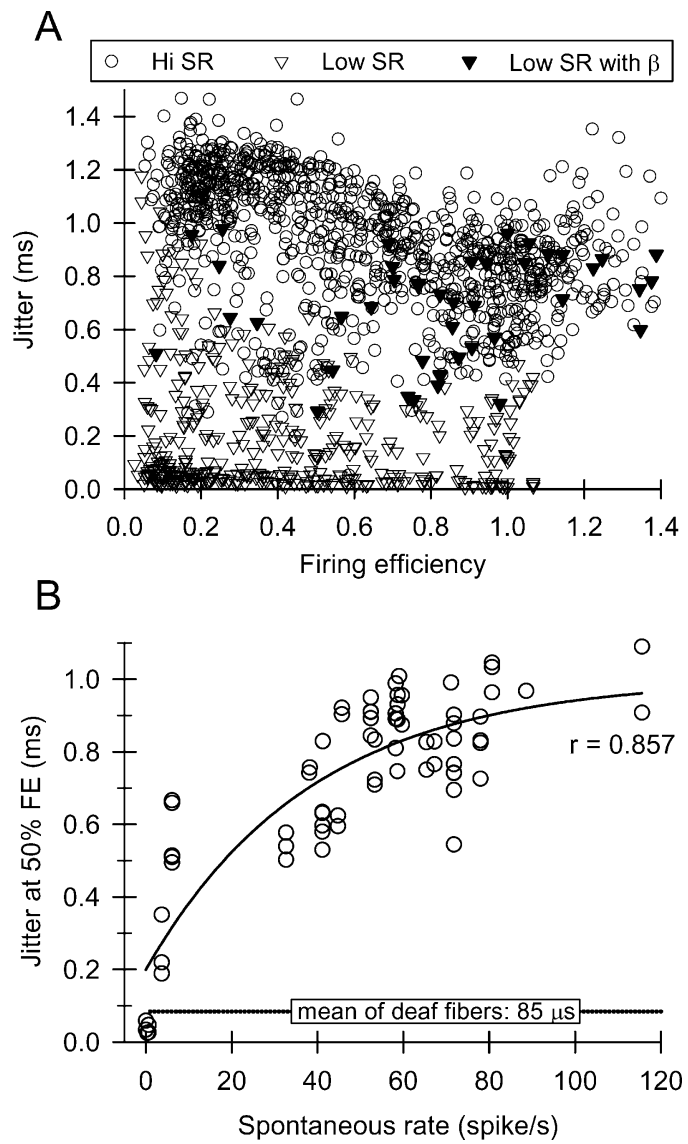

FIG. 9. (A) Temporal dispersion (jitter) plotted as a function of firing efficiency for 118 fibers. The symbol type indicates whether or not the fiber was a low- or high-SR fiber, and fibers in which an electrophonic response was evident. Firing efficiencies greater than 1 were observed in some fibers with high spontaneous rates and electrophonic responses. (B) Jitter at a firing efficiency of $50 \%$ plotted as a function of spontaneous rate. Also shown (by the horizontal line) is the mean jitter (again at an FE 50\%) for deaf fibers from cats of Miller et al. (2001b).

and high firing efficiencies. In those cases, adaptation was observed at mid levels that did not produce saturated response rates. In the other 10 cases, no adaptation was observed.

In some cases, the narrow bin widths of the upper plots are somewhat "misleading" to the extent that unsynchronized spikes are somewhat difficult to see. Thus, the slow increase in size of the peaks in the upper-left PST histogram reflects loss of the electrophonic response and, possibly, spontaneous activity. Similarly, the apparent rate decrement in the plot at the upper right does not reflect spike rate reductions, but rather the slow increase in jitter across the pulse train, as seen in the corresponding jitter plot.

Finally, it is relevant to note that one Figure 10 fiber (D48-4-2) had no measurable acoustic sensitivity or spontaneous activity. Nonetheless, the time-related increases in jitter observed at the highest stimulus level (Fig. 10, right column top jitter plot) were 
significantly greater than the jitter values reported in fibers of chemically deafened cats stimulated by single pulses (Miller et al. 1999, 2001b). Thus, a mechanism other than spontaneous or electrophonic activity may play a role in increasing jitter over repeated electric stimulation.

"Buildup" responses. Two unique response patterns were observed in many fibers across the duration of the electric pulse trains. One prominent pattern, which we call a "buildup" response, is seen to various degrees in the PST histograms of Figure 10. This pattern is characterized by increases in FE over as few as the first 2-3 pulses and as long as the first 10 pulses in the train. Short-duration buildup (over an 8-ms interval) is seen in the third column, top panel, of Figure 10, whereas longer buildup (20- to $30-\mathrm{ms}$ periods) is seen in the first and second columns for the lower stimulus levels. Although not particularly clear in the examples shown here, buildup patterns observed at the moderate levels would, in some cases, not persist at higher stimulus levels, suggesting an interaction with stimulus level. Finally, although "buildup" was observed in the fiber with no apparent acoustic sensitivity (Fig. 10, right column), as discussed later, this was uncommon.

Our data suggest that the incidence of "buildup" correlates with fiber hearing sensitivity. We surveyed all of the fibers' PST histograms for the presence of

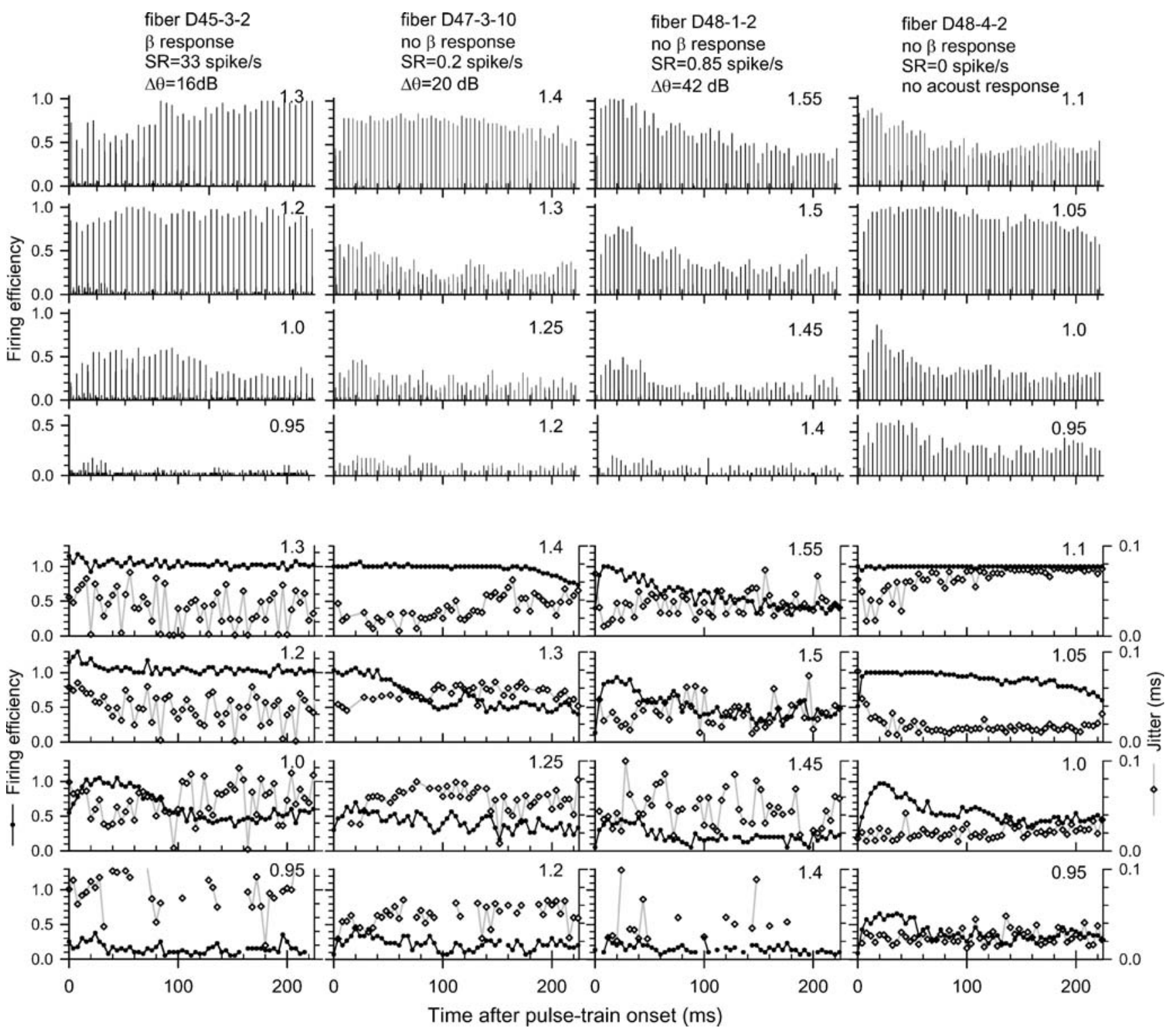

FIG. 10. PST histograms for four fibers, assessed at four stimulus levels each, using both narrow (50 $\mu \mathrm{s})$ bin widths (upper set of histograms) and wide (4 ms) bin widths (lower set of histograms). Also plotted on the lower set are the mean jitter values for each 4-ms bin. In that set, jitter data are plotted using open symbols, while filled symbols indicate FE values. 
the "buildup" pattern and assigned each fiber a 0 or 1 to denote the absence or presence of the buildup response. Figure 11 (lower panel), which plots this binary variable vs. acoustic threshold shift for 100 fibers, indicates that fibers with relatively low threshold shifts are more likely to produce this response. Using a 45- $\mathrm{dB}$ criterion threshold shift, fibers were divided into groups with either "low" or "high" threshold shifts and were found, via a $t$-test, to have significantly different incidences of the buildup pattern $\left(t=4.45, p_{\text {error }}<0.0001, d f=55\right)$.

It could be hypothesized that "buildup" is correlated with electrophonic or spontaneous activity. The inset plots of Figure 11 show the incidence of "buildup" in fibers with and without an electrophonic response (left inset) and in fibers with low and high SRs (right inset). There are trends toward greater incidence with the electrophonic response and high SR, although $t$-tests indicate only marginal significance $\left(\right.$ both $p_{\text {error }}=0.055$ ). We were unable to determine whether the electrophonic response or spontaneous activity was more strongly related to the buildup pattern. Inspection of the Figure 11 plots reveals that estimates of the overall incidence of "buildup" varied with the way in which the fiber population was sampled. Using the largest fiber sample of Figure 11 (i.e., data of the top left plot), "buildup" was found in $21 \%$ of fibers. However, by selecting a smaller population, 94 fibers that yielded data for at least three electric levels, "buildup" was observed in $53 \%$ of the fibers.

Bursting. We have observed a quasi-periodic pattern in many fibers that is characterized as sequences in which a fiber responds to each of several stimulus pulses, followed by periods over which the fiber fails
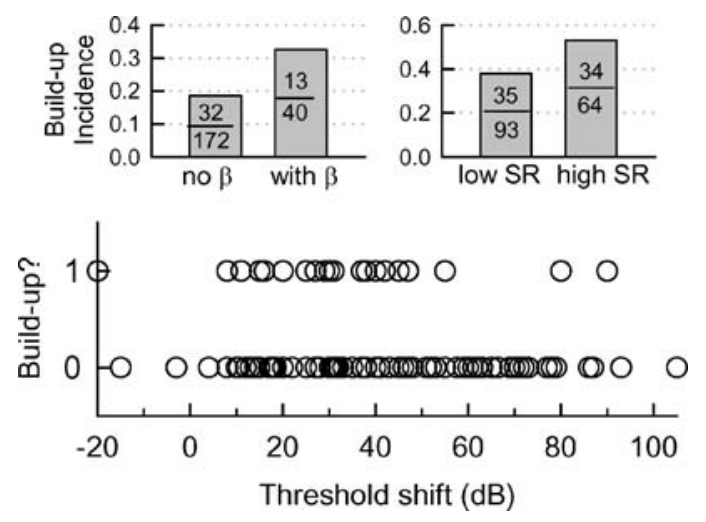

FIG. 11. Shown in the lower graph is a plot of a binary variable indicating the presence or absence of the "buildup" response as a function of acoustic threshold shift. Each circle indicates an individual fiber. The two upper graphs show the incidence of the buildup response as a function of the category (low, high) of spontaneous rate and as a function of the presence or absence of an electrophonic response. to respond to several sequential pulses. This pattern, typically described as "bursting," has been anecdotally reported by Shepherd and Javel (1997) and Litvak et al. (2003) in feline auditory nerve fiber response to electric pulse trains. Figure 12 shows dotraster plots from three bursting fibers stimulated at three levels. Several observations can be made. First, bursting can occur over a large portion of a fiber's dynamic range. Second, the extent of bursting can be reduced by increases in stimulus level, as clearly seen in the top histogram of fiber D45-3-2 (Fig. 12, middle column). Third, the data suggest that bursting may begin after periods of sustained firing, such that it may relate to an adapted state. It should be noted that bursting patterns were not correlated with the cat's respiration cycles or heart rate (which, in problematic cases, can modulate spike amplitude). Furthermore, cyclic spike amplitude modulations, typically seen when nerve pulsation is a problem, were not observed in these cases or were not observed to correlate with bursting periods.

The incidence of bursting was assessed in 53 fibers for which data were obtained for at least three stimulus levels and estimates of acoustic threshold were made. Of those, 35 fibers $(66 \%)$ were judged as exhibiting some degree of bursting. Again, using a 45- $\mathrm{dB}$ criterion for acoustic threshold shift, these fibers were divided into two groups: fibers with lowto-moderate and large threshold shifts. A $t$-test failed to find a significant difference in the incidence of this response pattern across the two groups $(t=0.77$, $\left.p_{\text {error }}=0.45, d f=51\right)$.

Bursting was further explored by assessing the length of each burst. Lengths of all spike sequences and spike-failure sequences were tabulated for seven fibers being evaluated, by visual assessment of dotraster plots, to exhibit bursting. Sequence lengths were computed by counting the number of interpulse intervals and therefore have a 4-ms resolution. Across the fibers, the median spike sequence length was 44 $\mathrm{ms}$ and the median spike-failure sequence length was $30 \mathrm{~ms}$. Histograms of lengths of the two sequence types are shown in Figure 13. Histograms for the spike-failure sequences have a peak at the shortest intervals, consistent with short spike failures that would not be categorized as a "burst," but rather, random failures. However, most of these spike-failure histograms exhibit a second peak that reflects bursting. In some cases (i.e., bottom three histograms), this second peak is prominent; in others, it is quite subtle. For the spike sequence histograms, two general contours are evident, one in which the probability of a sequence length decreases with length (e.g., top two or three plots) and another that exhibits a discernable mode, corresponding to a "characteristic" burst duration (e.g., lower graphs of Fig. 13). 

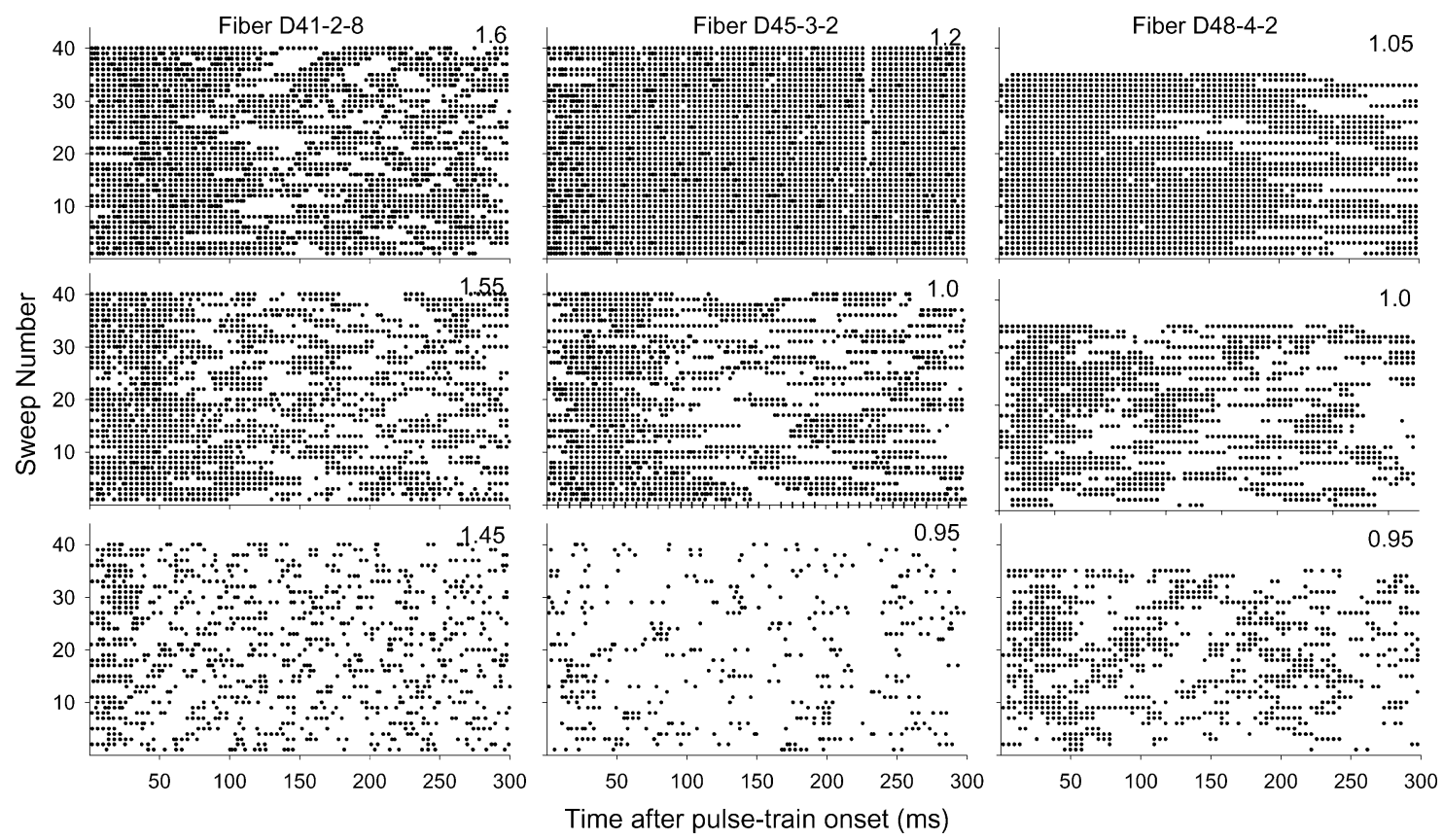

FIG. 12. Dot-raster plots of the responses to 300-ms pulse trains in three fibers exhibiting "bursting" patterns. The plots obtained at three different stimulus levels are shown in each column, with the current levels indicated in the top right corner of each plot.

As noted above, bursting was not locked to respiratory or cardiac patterns. Our stimulus protocol (of sequential stimulation by an electric train, an acoustic noise burst, and combined electric and acoustic stimuli) provided a useful control condition, the acoustic-alone condition, to check for such artifactual responses. Figure 14 shows dot-raster plots for a fiber in response to the three stimuli, demonstrating a consistent finding: a lack of bursting responses to the acoustic noise. The plots also reveal a unique and intriguing response pattern-the cessation of bursting following the acoustic noise. In considering that phenomenon, recall that the three stimuli were presented in interleaved order. Thus, the plots effectively present repeated measures of response property.

\section{DISCUSSION}

The animal model described in this study exhibited moderate $(20-30 \mathrm{~dB})$ hearing losses, presumably as a result of trauma from the cochleostomy and electrode insertion. Initial losses were as low as $5-10 \mathrm{~dB}$; subsequent losses occurred at rates as low as $1.3 \mathrm{~dB} / \mathrm{h}$. Spontaneous activity was evident, although the SR distribution was skewed to below normal rates, consistent with cochlear trauma (Liberman and Dodds 1984). It is not clear what caused the slow threshold increases over time. Cochlear insults may have promoted slow changes in ionic concentrations in the scalae, thereby reducing interscalar potentials and
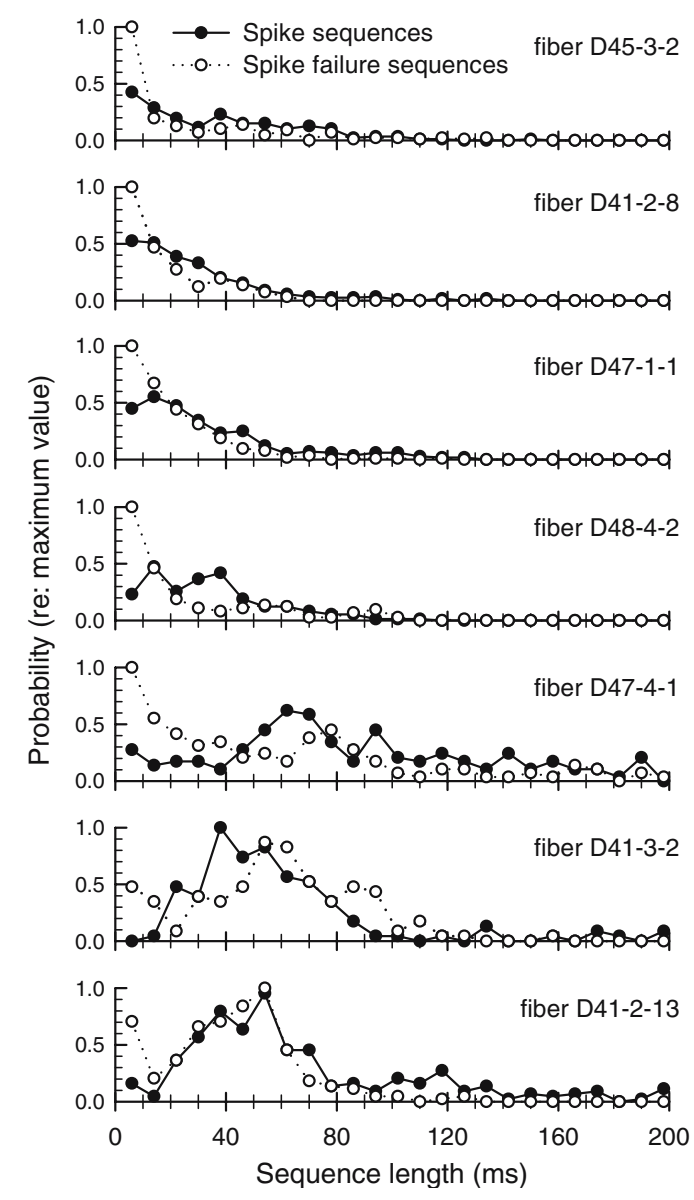

FIG. 13. Histograms of the length of each sequence of spikes (filled symbols) and the length of each sequence of spike failures (open symbols) are shown for seven fibers that exhibited bursting. 


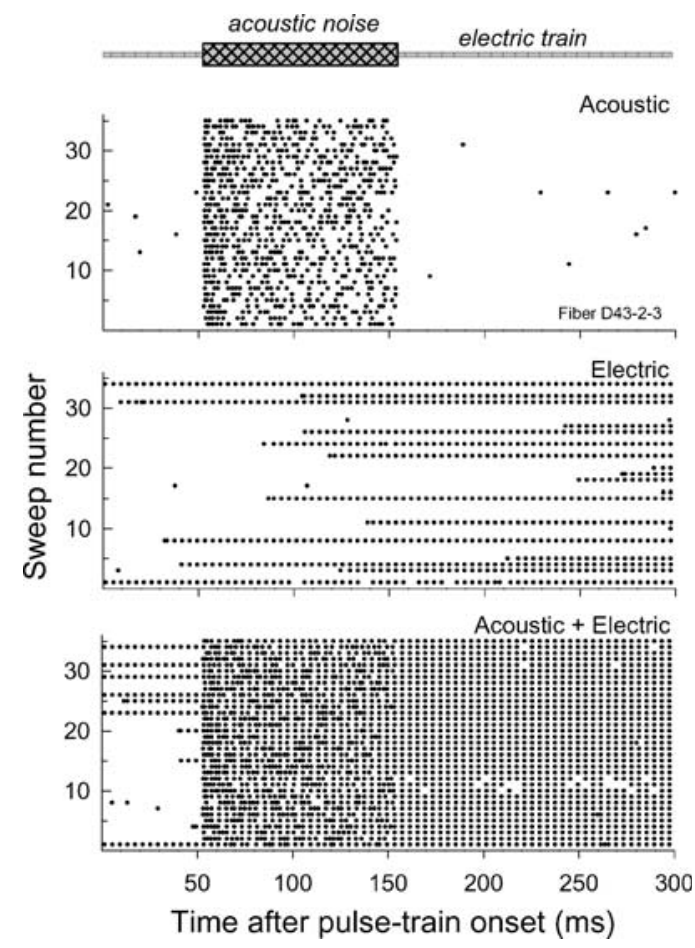

FIG. 14. Dot-raster plots of a fiber exhibiting bursting. Three different stimuli were used. In the top plot, a 96-dB SPL wideband noise stimulus was presented for $100 \mathrm{~ms}$, beginning at $50 \mathrm{~ms}$. In the middle plot, the standard 250 pulse/s electric train was presented. In the bottom plot, both of the above stimuli were presented together. In that plot, note the loss of the bursting pattern following offset of the acoustic stimulus.

acoustic sensitivity. It is unlikely that general changes in physiological status led to reduced sensitivity, as several animal vital signs were maintained within normal limits throughout the experiments.

\section{Differences in the responses of hearing and deaf fibers}

Spontaneous activity has been hypothesized to reduce electric sensitivity relative to that of deaf and silent fibers. Earlier gross potential (ECAP) work found that threshold and dynamic range were, relative to hearing ears, reduced in deaf ears ( $\mathrm{Hu}$ et al. 2003). Spontaneous activity will put fibers in states of partial refractoriness, thus elevating threshold. It could also induce adaptation-like changes that could make it more difficult to achieve "saturated" responses, thereby increasing dynamic range. Our data provide such evidence by showing a mean $(3 \mathrm{~dB})$ increase in threshold and a small $(<1 \mathrm{~dB})$ increase in dynamic range relative to deaf fiber data. With a range of SRs, acoustically sensitive nerves would have greater ranges of fiber thresholds, further increasing the whole nerve's dynamic range. There was no evidence that a "stochastic resonance"-type response was created by spontaneous activity.
Jitter was greatly elevated relative to deaf-fiber values and was related to SR. Increases were likely attributable not only to the addition of random spike activity, but also to the alteration of membrane states, as spontaneous activity would cause partial refractoriness. Partially refractory fibers have greater variance in their electric thresholds (Dynes 1996), which could contribute to greater jitter and ensemble dynamic range. The neural representation of electric stimuli in ears with residual hearing may therefore be expected to be more "natural" (less deterministic) than what is produced by deaf ears. The peripheral processes of auditory nerve fibers are a proposed source of membrane noise and jitter (van den Honert and Stypulkowski 1984), and these processes are vulnerable to chemical deafening (Leake-Jones et al. 1982). However, it is not clear how relevant these factors are to our comparison of deaf and hearing fibers, as our previous work with acutely deafened ears suggests that peripheral processes are functional in those cases (Miller et al. 1999, 2003). Finally, it is hypothesized that ongoing spike activity deactivates some voltage-gated ion channels at the nodes of Ranvier, thereby increasing jitter (Verveen 1961; Rubinstein 1995). Data from a fiber without spontaneous activity or acoustic sensitivity (Fig. 10, right column) provide corroborating evidence. Across the stimulus duration, jitter substantially increased, consistent with the idea that membrane noise increases during sustained firing.

The $\beta$ (electrophonic) response markedly influenced the electric responses. It demonstrated fast adaptation to our $250 \mathrm{pulse} / \mathrm{s}$ electric stimulus (mean $\tau=18 \mathrm{~ms}$ ), whereas the $\alpha$ response showed little, if any, adaptation. Such differential adaptation may be perceptually important, as temporal and spectral representations of a stimulus would undergo large changes over periods relevant to individual speech tokens and formant transitions. The spectra of Figure 7 show that $\beta$ responses greatly reduced the harmonic content of the spike code. $\beta$ adaptation was also strongly dependent upon level. Low levels could evoke sustained $\beta$ responses, whereas higher levels induced fast adaptation.

It is not clear that $\beta$ responses are relevant to currently implanted individuals with residual hearing. The Iowa Cochlear Implant Center has collected ECAP responses from several implantees with residual acoustic sensitivity in the implanted ear. Preliminary results do not indicate $\beta$ responses in their ECAP recordings (Abbas et al. 2005), which would be expected to appear as a second biphasic CAP-like waveform occurring 2-3 ms after the ECAP. Given the typical audiogram of these users (i.e., large losses at high and moderate frequencies), these negative findings may be expected, as electromotile activation 
of the basal part of the cochlea may be needed for $\beta$ responses. However, unpublished data collected by the first author in collaboration with Dr. Bryan Pfingst indicate that a chronically implanted animal can produce $\beta$ responses. In this case, $\beta$ responses were recorded from a guinea pig implanted 14 days earlier with an electrode array that extended several millimeters into the cochlea. Longitudinal data from chronically implanted animals would be useful in resolving the role of the $\beta$ response in implanted ears.

\section{Adaptation}

Adaptation to our 250 pulse/s trains differs from that observed in response to acoustic stimuli. PST histograms to acoustic stimuli demonstrate a stereotypic shape characterized by (1) fast, initial, adaptation, with a time constant of 1-10 $\mathrm{ms}$ and (2) slower, subsequent, adaptation, with a time constant of 20$100 \mathrm{~ms}$. Both components are observed for wide ranges of acoustic levels, including those producing "saturated" responses (Kiang et al. 1965; Westerman and Smith 1984). This differs from the patterns reported here. Mild electric adaptation occurred at levels evoking FEs in the mid-to-upper part of the fiber's dynamic range, whereas little adaptation occurred at lower FEs. Furthermore, adaptation could be "overcome" by stimulating a fiber at high levels (cf. Figs. 10 and 12), something not observed with acoustic stimulation. Javel (1990) noted that adaptation to electric stimuli calls for a reevaluation of the mechanisms of adaptation, which is often solely attributed to the hair cell and synapse (e.g., Smith and Brachman 1982; Meddis 1986). One approach to this issue would be to compare acoustic and electric adaptation as functions of instantaneous, or short-term, response rates. Such comparisons may demonstrate that acoustic rates are limited to ranges lower than those possible with electric excitation and that "membrane adaptation" is not achieved with acoustic stimulation.

The modest $\alpha$ adaptation is consistent with a report of only minor ECAP adaptation to 250 pulse/s trains presented to chemically deafened animals (Matsuoka et al. 2000). Approximately 15\% of our fibers exhibited large degrees of adaptation. It is not clear what underlies those shifts, although sudden reductions in sensitivity to electric pulses have been reported in $10 \%$ of fibers from deafened animals stimulated with low-rate (33.3 pulse/s) trains (Miller et al. 1999).

\section{Buildup responses}

"Buildup" in response to 250 pulse/s trains has been observed in the ECAPs of hearing ears (Miller et al.
2005), suggesting that it can be a robust phenomenon. ECAP "buildup" has not been reported in deaf ears. In their deaf animals, Matsuoka et al. (2000) did not observe increases in ECAP amplitude from the first to the second pulse, using pulse trains presented at various rates. Haenggeli et al. (1998) examined ECAPs in deafened and hearing rats and reported amplitude vs. pulse number functions that generally decreased monotonically. In our study, "buildup" was correlated with hearing sensitivity; fibers with larger threshold shifts were less likely to exhibit it. "Buildup" was also weakly correlated with the presence of $\beta$ responses and SR. Using a large pool of fibers, we observed "buildup" in $21 \%$ of 212 fibers. However, "buildup" was dependent on stimulus level, which may explain greater "buildup" incidence $(53 \%)$ when only fibers with data at three or more levels were considered. At any rate, we can conclude that "buildup" occurred in a large minority of fibers.

A simple interpretation of "buildup" is that it reflects across-pulse integration. Previous estimates of integration time constants for feline fibers have been in the range of 150-500 $\mu$ s (Dynes 1996; Cartee et al. 2000), presumably too short to account for the slow buildup reported here. Those earlier estimates were obtained in deafened cochleae that may have undergone some neural degeneration. If so, the relatively intact peripheral processes of our preparations may have played a greater role in the excitation process, possibly introducing a relatively slow integrative process.

Other factors may have contributed to buildup responses. Pulse train stimuli may result in adaptation of spontaneous activity, which could, in turn, reduce a fiber's electric threshold. As noted above, we found a weak correlation between "buildup" and spontaneous rate. A weak correlation existed between buildup responses and $\beta$ activity. Fast $\beta$ adaptation could result in lower electric thresholds at the 250 pulse/s rate of our stimuli, because, at that rate, $\beta$ responses would act (in concert with refractoriness) as competing stimuli. However, "buildup" was also observed in fibers without $\beta$ responses. Perhaps low or "subthreshold" levels of neurotransmitter release may also play a role.

\section{Bursting}

Bursting in response to repetitive electric stimuli has been noted by others (Shepherd and Javel 1997; Litvak et al. 2003). Litvak et al. (2003) identified two major classes of spike interval histograms (IHs) based on whether or not the tail of the histogram (i.e., the region of relatively long intervals) was described by a decaying exponential function. They categorized $48 \%$ of their histograms as "type II," i.e., having nonexponential tails. This class of histograms included those 
with a clearly identifiable mode that corresponded to bursting. The notion of two classes of fibers based on a Poisson-like process (i.e., a decaying exponential IH) and those with a nonexponential tail is consistent with the two shapes described in our histograms of Figure 13. In one fiber with clear bursting, Litvak et al. (2003) reported a mode in the $\mathrm{IH}$ at $60 \mathrm{~ms}$ - a preferred interval not inconsistent with the modes shown in Figure 13. Shepherd and Javel (1997) did not quantify bursting responses; however, their exemplar PST histograms of bursting (i.e., their Fig. 19) suggest similarities and differences with the bursting reported here. First, both their fiber and our examples demonstrated a tendency for bursting to give way to sustained firing with increases in stimulus level. However, their data include bursting over much longer intervals, on the order of a few seconds.

The mechanism of bursting is not known. Shepherd and Javel (1997) suggested that it is attributable to neural degeneration. In addition to bursting of driven activity, they reported it in the spontaneous firing of fibers from presumably deaf ears. Noting that the IHs of this spontaneous activity were different from those of a "normal" cat (i.e., one not chemically treated), and that bursting was not observed in any fibers from their normal cat, they concluded that bursting was indicative of pathology. The results of our study, however, do not support that conclusion, as the cochleae of our cats were not chemically treated and acoustically sensitive fibers exhibited bursting to electric stimuli. Notably, we never observed bursting in spontaneous firing patterns or burst intervals on the order of a second. Thus, the bursting of Shepherd and Javel (1997) may have been attributable to mechanisms not found in our preparations. From the results in our study as well as those that used chemically deafened and chronically deaf cats, it appears that bursting is caused by repetitive and sustained electrical stimulation of nerve fibers. If that is the case, then bursting phenomena could be explored with computer modeling approaches. Such models may have to account for long-term metabolic effects or homeostatic factors that are typically not incorporated in computer simulations. It is also possible that bursting occurs in the human auditory nerve in response to repetitive electrical stimulation, something that is quite characteristic of the stimuli used in present-day cochlear implant devices. The relevance of this phenomenon to perception is not clear.

\section{ACKNOWLEDGMENT}

This research was funded by the United States National Institutes of Health (Contract N01-DC-2-1005).

\section{REFERENCES}

Abbas PJ, Etler CP, Nourski K, Brown CJ, Miller CA. Responses to electrical stimulation in individuals with residual hair cell function. Abstract. Objective Measures in Cochlear Implants. Germany, Hannover, 2005.

BeVIngton PR. Data reduction and error analysis for the physical sciences. New York, McGraw-Hill, 1969.

Brownell WE, Bader CR, Bertrand D, de Ribaupierre Y. Evoked mechanical responses of isolated cochlear outer hair cells. Science 227:194-196, 1985.

Cartee LA, van den Honert C, Finley CC, Miller RL. Evaluation of a model of the cochlear neural membrane. I. Physiological measurement of membrane characteristics in response to intrametal electrical stimulation. Hear. Res. 146:143-152, 2000.

DyNEs SBC. Discharge characteristics of auditory nerve fibers for pulsatile electrical stimuli. Ph.D. thesis, Massachusetts Institute of Technology (Dept. of Physics), 1996.

Gantz BJ, Turner CW. Combining acoustic and electrical hearing. Laryngoscope 113:1726-1730, 2003.

Haenggeli A, Zhang JS, Vischer MW, Pelizzone M, Rouiller EM. Electrically evoked compound action potential (ECAP) of the cochlear nerve in response to pulsatile electrical stimulation of the cochlea in the rat: effects of stimulation at high rates. Audiology 37:353-371, 1998.

Hu N, Abbas PJ, Miller CA, Robinson BK, Nourski KV, Jeng FC, Abkes BA, Nichols JM. Auditory response to intracochlear electric stimuli following furosemide treatment. Hear. Res. 185:77-89, 2003.

JAVEL E. Acoustic and electrical encoding of temporal information. In: Miller JM and Spelman FA (eds) Cochlear implants. Models of the Electrically Stimulated Ear. New York, Springer Verlag, pp 247-295, 1990.

JAVEL E, SHEPHERD RK. Electrical stimulation of the auditory nerve. III. Response initiation sites and temporal fine structure. Hear. Res. 140:45-76, 2000.

Kiang NY, Watanabe T, Thomas EC, Clark LF. Discharge Patterns of Single Fibers in the Cat's Auditory Nerve.Cambridge, MIT Press, 1965.

Kiefer J, von Ilberg C, Reimer B, Knecht R, Gall V, Diller G, Sturzebecher E, Pfennigdorff T, Spelsberg A. Results of cochlear implantation in patients with severe to profound hearing lossimplications for patient selection. Audiology 37:382-395, 1998.

Leake-Jones PA, Vivion MC, O'Reilly BF, Merzenich MM. Deaf animal models for studies of a multichannel cochlear prosthesis. Hear. Res. 8:225-246, 1982.

LiBERMAN MC. Auditory-nerve response from cats raised in a lownoise chamber. J. Acoust. Soc. Am. 63:442-455, 1978.

LiBERMAN MC. The cochlear frequency map for the cat: labeling auditory nerve fibers of known characteristic frequency. J. Acoust. Soc. Am. 72:1441-1449, 1982.

Liberman MC, DodDs LW. Single-neuron labeling and chronic cochlear pathology. II. Stereocilia damage and alterations of spontaneous discharge rates. Hear. Res. 16:43-53, 1984.

Liberman MC, Kiang NY. Acoustic trauma in cats. Cochlear pathology and auditory-nerve activity. Acta Oto-laryngol., Suppl. 358:1-63, 1978.

Litvak LM, Smith ZM, Delgutte B, Eddington DK. Desynchronization of electrically evoked auditory-nerve activity by highfrequency pulse trains of long duration. J. Acoust. Soc. Am. 114:2066-2078, 2003.

Matsuoka AJ, Abbas PJ, Rubinstein JT, Miller CA. The neuronal response to electrical constant-amplitude pulse train stimulation: evoked compound action potential recordings. Hear. Res. 149:115-128, 2000.

MEDDIS R. Simulation of mechanical to neural transduction in the auditory receptor. J. Acoust. Soc. Am. 79:702-711, 1986. 
Miller CA, Abbas PJ, Robinson BK, Rubinstein JT, Matsuoka AJ. Electrically evoked single-fiber action potentials from cat: responses to monopolar, monophasic stimulation. Hear. Res. 130:197-218, 1999.

Miller CA, Abbas PJ, Robinson BK. Response properties of the refractory auditory nerve fiber. J. Assoc. Res. Otolaryngol. 2:216-232, 2001a.

Miller CA, Robinson BK, Rubinstein JT, Abbas PJ, Runge-Samuelson CL. Auditory nerve responses to monophasic and biphasic electric stimuli. Hear. Res. 151:79-94, 2001b.

Miller CA, Abbas PJ, Nourski KV, Hu N, Robinson BK. Electrode configuration influences action potential initiation site and ensemble stochastic response properties. Hear. Res. 175:200214, 2003.

Miller CA, Robinson BK, Abbas PJ, Nourski KV, Jeng FC. Effects of remaining hair cells on cochlear implant function. 12th Quarterly Progress Report. NIH Contract N01-DC-2-1005, 2005.

Moxon EC. Neural and mechanical responses to electric stimulation of the cat's inner ear.Ph.D. thesis, Massachusetts Institute of Technology (Dept. of Electrical Engineering), 1971.

Ni D, Shepherd RK, Seldon HL, Xu SA, Clark GM, Millard RE. Cochlear pathology following chronic electrical stimulation of the auditory nerve. I. Normal hearing kittens. Hear. Res. 62: 63-81, 1992.

Nourski KV, Abbas PJ, Miller CA, Robinson BK, Jeng FC. Effects of acoustic noise on the auditory nerve compound action potentials evoked by electric pulse trains. Hear. Res. 202:141-153, 2005.
Nuttall AL, Ren T. Electromotile hearing: evidence from basilar membrane motion and otoacoustic emissions. Hear. Res. 92:170-177, 1995.

Rubinstein JT. Threshold fluctuations in an N sodium channel model of the node of Ranvier. Biophys. J. 68:779-785, 1995.

ShePherd RK, Javel E. Electrical stimulation of the auditory nerve. I. Correlation of physiological responses with cochlear status. Hear. Res. 108:112-144, 1997.

Smith RL, Brachman ML. Adaptation in auditory-nerve fibers: a revised model. Biol. Cybern. 44:107-120, 1982.

Van Den Honert C, Stypulkowski PH. Physiological properties of the electrically stimulated auditory nerve. II. Single fiber recordings. Hear. Res. 14:225-243, 1984.

Verveen AA. Fluctuation in excitability. Amsterdam, Drukkerij Holland, 1961.

Von Ilberg C, Kiefer J, Tillein J, Pfenningdorff T, Hartmann R, Sturzebecher E, Kuinke R. Electric-acoustic stimulation of the auditory system. New technology for severe hearing loss. ORL J. Otorhinolaryngol. Relat. Spec. 61:334-340, 1999.

WARR WB, GUINAN JJ JR. Efferent innervation of the organ of corti: two separate systems. Brain Res. 173:152-155, 1979.

Westerman LA, Smith RL. Rapid and short-term adaptation in auditory nerve responses. Hear. Res. 15:249-260, 1984.

Xu J, Shepherd RK, Millard RE, Clark GM. Chronic electrical stimulation of the auditory nerve at high stimulus rates: a physiological and histopathological study. Hear. Res. 105: 1-29, 1997. 